\title{
Finalement rien ne se passe jamais comme on l'avait imaginé
}

\section{Christoph Bosshard}

Dr, vice-président de la FMH, responsable du département Données, démographie et qualité

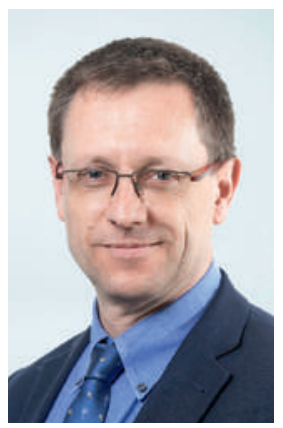

Qui de nous pourrait affirmer avec la main sur le cœur qu'il a toujours su où la vie allait le mener? Si nos souhaits personnels et les valeurs que nous prônons déterminent une grande partie de nos choix, ils évoluent aussi au fil du temps. De plus, savoir comment et où fixer les priorités et jusqu'où faire des concessions diffère d'une personne et d'une situation à l'autre et peut changer avec le temps.

Alors qu'il y a 30 ans, on déconseillait aux jeunes de se lancer dans des études de médecine en invoquant une surabondance de médecins, la situation d'aujourd'hui s'est totalement inversée. Pour couvrir le besoin de praticiens en Suisse, nous sommes obligés de faire appel à des collègues étrangers. Il n'est pas rare d'ailleurs que les médias rappellent que trop de médecins tourneraient le dos à leur profession après une formation coûteuse. De plus, la tendance actuelle en faveur du travail à temps partiel gagne aussi le corps médical. Dans ce contexte, la FMH et l'ASMAC ont voulu disposer de données fondées sur le nombre effectif de médecins qui n'exercent plus auprès des patients et connaître les raisons de leur réorientation professionnelle, et ont donc décidé de mandater une étude dont les résultats sont présentés à la page 1132 de ce numéro. Si, jusqu'à présent, il a été fait état d'un nombre important de médecins qui quitteraient leur profession, le résultat de l'enquête laisse plutôt songeur. En effet, ils ne sont environ que $10 \%$ de l'ensemble des diplômés

\section{Il faut créer des modèles de travail permettant de concilier vie professionnelle et vie privée.}

annuels en médecine humaine à abandonner la profession avant l'âge de la retraite. La question de la pénurie de médecins ne se réglera donc pas par une obligation de pratique après la formation mais bien plus par la création de places d'études supplémentaires en médecine humaine. Les fonds de la Confédération, prévus dans le message relatif à l'encouragement de la formation, de la recherche et de l'innovation pour les années 2017 à 2020, sont donc absolument nécessaires. Par ailleurs, les compétences médicales ne servent pas uniquement au chevet du patient mais aussi dans l'administration et les milieux économiques, où l'industrie pharmaceutique, les technologies médicales tout comme les caisses-maladie et les assurances ont besoin de médecins au bénéfice d'une formation et d'expérience pratique. Si on enlève ces domaines d'activité aux 10\% mentionnés, la capacité à contrer la pénurie de médecins en faisant revenir nos collègues à leur vocation initiale se réduit encore davantage. L'étude relève que seul un médecin sur dix qui n'exerce plus peut s'imaginer reprendre son activité auprès des patients.

Dans ce contexte, les mesures demandées par la FMH et l'ASMAC revêtent encore plus d'importance pour que chaque médecin puisse accorder le plus de temps possible à ses patients. La recrudescence constante des

\section{S'agissant de la pénurie de médecins,} une augmentation des places d'études est inévitable.

tâches administratives, telles que le démontrent les études concomitantes à l'introduction de SwissDRG, met en évidence un champ d'action clair. Des idées et solutions existent depuis longtemps mais pour une institution, gérer un secrétariat supplémentaire coûte de l'argent, et les médecins-assistants ont été pendant longtemps une main d'œuvre bien trop bon marché à la disponibilité illimitée. Ce n'est que sous la pression de la loi sur le travail qu'une évolution a vu le jour, alors que la situation était bloquée depuis plus de dix ans. Ce potentiel d'amélioration n'a pas encore été épuisé. Celui qui ignore la tendance au travail à temps partiel de notre société fait le jeu de l'autruche et s'enfonce la tête dans le sable. Il s'agit en effet de proposer des modèles de travail susceptibles d'offrir la marge de manœuvre nécessaire pour concilier travail auprès des patients avec famille, vie de couple ou autres priorités individuelles.

Si nous voulons être prêts pour les enjeux de demain, nous devons tirer les leçons de ces résultats et mettre en œuvre les conclusions qui en découlent. Je suis persuadé que cela augmentera la reconnaissance et aussi la satisfaction des médecins, et aura un impact direct sur la motivation, les bénéfices pour le patient et sur l'efficacité. 